\title{
A photometric long-term study of chemically peculiar stars in open clusters ${ }^{\star}$
}

\author{
E. Paunzen ${ }^{1}$, H. Hensberge ${ }^{2}$, H. M. Maitzen ${ }^{1}$, M. Netopi ${ }^{1,3}$, C. Trigilio ${ }^{4}$, L. Fossati ${ }^{1,5}$, U. Heiter ${ }^{6}$, and M. Pranka ${ }^{1}$ \\ ${ }^{1}$ Institut für Astronomie der Universität Wien, Türkenschanzstr. 17, 1180 Wien, Austria \\ e-mail: Ernst.Paunzen@univie.ac.at \\ 2 Royal Observatory of Belgium, Ringlaan 3, Brussels, Belgium \\ ${ }^{3}$ Hvar Observatory, Faculty of Geodesy, University of Zagreb, Kačićeva 26, 10000 Zagreb, Croatia \\ ${ }^{4}$ INAF - Osservatorio Astrofisico di Catania, via S. Sofia 78, 95123 Catania, Italy \\ 5 Department of Physics and Astronomy, The Open University, Milton Keynes, MK7 6AA, UK \\ ${ }^{6}$ Department of Physics and Astronomy, Uppsala University, Box 516, 75120 Uppsala, Sweden
}

Received 2 December 2009 / Accepted 11 September 2010

\section{ABSTRACT}

\begin{abstract}
Context. Photometric variability of chemically peculiar (CP) stars of the upper main sequence is closely connected to their local stellar magnetic field and their rotational period. Long term investigations, as presented here, help us to identify possible stellar cycles (as in the Sun). Furthermore, these data provide a basis for detailed surface mapping techniques.

Aims. Photoelectric Strömgren uvby time series for $27 \mathrm{CP}$ stars within the boundaries of open clusters are presented. In addition, Hipparcos photometric data (from 1989 to 1993) are used for our analysis. Our observations cover a time period of about six years (1986 to 1992) with typically fifteen measurements for each objects. These observations help us to determine the rotational periods of these objects.

Methods. A standard reduction procedure was applied to the data. When possible, we merged our data sets with already published ones to obtain a more significant result. A detailed time series analysis was performed, involving five different methods to minimize spurious detections.

Results. We established, for the first time, variability for fourteen CP stars. For additional two stars, a merging of already published data sets, resulted in more precise periods, whereas for six objects, the published periods could be confirmed. Last, but not least, no significant variations were found for five stars. Apart from six stars, all targets seem to be members of their host open clusters.

Conclusions. The present observations fill an important gap in previous photometric long-time studies of CP stars. The presented open cluster members are excellent targets for follow-up observations, employing for example polarimetric, high-resolution spectroscopic, and surface mapping techniques.
\end{abstract}

Key words. stars: chemically peculiar - open clusters and associations: general - stars: variables: general

\section{Introduction}

The group of chemically peculiar $(\mathrm{CP})$ stars on the upper main sequence display peculiar lines and line strengths, in addition to other peculiar features such as a strong global stellar magnetic field (Babcock 1947). This subclass of B to F-type stars is characterized by variable line strengths and radial velocity changes as well as photometric variability of in general the same periodicity. One can usually distinguish between He-weak/strong, $\mathrm{HgMn}, \mathrm{Si}, \mathrm{SrCrEu}$, and Am stars. The subgroup of SrCrEu objects, for example, typically have overabundances of up to several dex for e.g., Sr, Cr, Eu, and other rare earth elements compared to the Sun.

Photometric variability of the CP star $\alpha^{2} \mathrm{CVn}$ was first reported by Guthnik \& Prager (1914). The light curves can be fitted well by a sine wave and its first harmonic with varying amplitudes for different photometric filter systems. For some CP stars, a double-wave structure of the photometric light curves

* All photometric measurements are available in electronic form at the CDS via anonymous ftp to

cdsarc.u-strasbg.fr (130.79.128.5) or via

http://cdsarc.u-strasbg.fr/viz-bin/qcat?]/A+A/525/A16 is detected (Maitzen 1980). However, similar magnetic field modulus variations are rare exceptions (Mathys et al. 1997).

The variability of $\mathrm{CP}$ stars is explained in terms of the oblique rotator model (Stibbs 1950), according to which, the period of the observed light, spectrum, and magnetic field variations is the rotational period. Accurate knowledge of the period of variability and its evolution in time for $\mathrm{CP}$ stars is a fundamental step in understanding their complex behaviour, especially as far as it concerns the phase relation between the magnetic, spectral, and light variations (Mikulášek et al. 2010).

In 2004, an on-line catalogue of photometric observations of magnetic CP stars $\left(\mathrm{mCPod}^{1}\right)$ was initiated (Mikulášek et al. 2007). This was intended to gather all available photometric data for CP stars, into a single, freely accessible database. The archive presently contains about 150000 individual measurements of $151 \mathrm{CP}$ stars and is being constantly updated with the latest photometric data.

In this paper, we present Strömgren uvby time series for $27 \mathrm{CP}$ stars within the boundaries of open clusters. In addition, we queried the Hipparcos photometric database (van Leeuwen et al. 1997) for entries of our targets and found four matches.

1 http://astro.physics.muni.cz/mcpod/ 
Our observations cover a time interval of about six years with typically fifteen measurements for each object. A detailed time series analysis was performed, implementing five different methods to minimize spurious detections.

Because the variability is assumed to be related to the rotation, a detailed study of the light curves is essential, not only to determine astrophysical parameters using more realistic stellar model atmospheres, but also to interpret the substantial information provided when mapping the stellar surface with Doppler imaging techniques (Lehmann et al. 2007). The choice of open cluster CP stars allows us to establish fundamental parameters such as the age, distance, reddening, and metallicity of numerous cluster members more accurately than usually possible for Galactic field stars.

\section{Target selection, observations, and reduction}

This work was initiated by the European working group on chemically peculiar stars of the upper main sequence (Mathys et al. 1989). It continues and complements an extensive survey performed by North et al. (1992). A continuation of these efforts had already been initialized using the Rapid Eye Mount (REM) telescope at La Silla (P.I.: L. Fossati).

The targets are established as candidate CP stars in open clusters mainly by the extensive photoelectric $\Delta a$ survey (e.g. Maitzen 1993). The comparison stars were chosen in the vicinity of the targets within the same magnitude range (if possible). Table 1 lists the target and comparison stars and their WEBDA $^{2}$ numbers. The data for the $V$ magnitude, spectral type, and $\Delta a$ measurement (Maitzen 1976) were also taken from WEBDA.

The star CP-60944 is a close visual binary with almost equally bright components. In the literature, there is a constant confusion about the designation of this object. Renson \& Manfroid (2009) list two entries in their most recent catalogue of Am and Ap stars, with $V$-magnitudes of 8.3 and 8.8, respectively. Both components are classified as B8 Si stars therein. The published $\Delta a$ value (Maitzen \& Hensberge 1981) is a combined value of both components, since it was impossible to separate them.

In addition, we queried the Hipparcos photometric database (van Leeuwen et al. 1997) for entries of the photoelectrically observed targets. In total, we found four matching entries: HD 56343, HD 87405, HD 89856, and HD 127924.

The photometric observations in the Strömgren uvby system were performed from 1986 to 1992 at the $50 \mathrm{~cm}$ Danish Telescope (SAT) and with the $61 \mathrm{~cm}$ Bochum telescope at LaSilla. The Bochum telescope was equipped with a dry-icecooled EMI 9502 photomultiplier, whereas the SAT contains an EMI 9789QB one. A detailed log of observations is given in Table 2.

All data were corrected for atmospheric extinction and sky background. In a first step, the standard extinction coefficients for LaSilla of the individual filters were used. For most of the nights, this choice was valid and very effective. For the observing nights in 1991 and 1992, the Volcano eruption of Pinatubo, we had to recalculate the coefficients as listed in Table 2. Finally, all observations were corrected to a heliocentric standard time frame.

All photometric measurements are available in electronic form at the CDS, from the first author and will be included in mCPod.

\footnotetext{
${ }^{2}$ http://www. univie.ac.at/webda
}

\section{Time series analysis}

The time series analysis of unevenly spaced data for a wide variety of time frames is not straightforward. Several effects (for example aliasing) severely influence the corresponding diagnostic diagrams.

To minimize the effects introduced by the limitations of the data sets and determine a stable solution, we applied five different time series analysis methods:

- Lafler - Kinman method (Lafler \& Kinman 1981);

- modified Lafler - Kinman method (Hensberge et al. 1977);

- phase-dispersion Method (Stellingwerf 1978);

- Renson method (Renson 1978);

- standard Fourier method (Deeming 1975).

The first of the four aforementioned methods are optimized to analyse sparsely distributed data sets. The results of the different methods were compared and the significant periods extracted. The complete set of results and published ones are listed in Table 1. The derived periods are significant to the last given digit. All computations were performed using the programme package Peranso ${ }^{3}$.

The decision about the (non-)variability of the individual objects was made after applying the above-mentioned time-series analysis methods. If the detection limit of all four algorithms failed, we assigned an object "constant" within the precision of our measurements. The definition of these limits can be found in the specific references and are mainly based on the appearance of significant peaks in the amplitude-frequency domain.

Figure 1 shows the phase diagrams of all programme stars with a well-established period in differential Strömgren uvby. For the folding process, the periods listed in Table 1 are used. The light curves of almost all targets are in phase for the four used filters. However, the $y$ data for NGC 2516 \#26 and NGC $3532 \# 449$, as well as the $v$ data for NGC $5460 \# 142$ are not in phase with those of the other filters. The light curves of NGC 2516 \#230 and NGC 6281 \#15 are very asymmetric, especially for the $u$ filter. The shape of the light curve for NGC 6281 \#15 is indicative of a double wave. These two stars certainly require further attention and complementary data to shed more light on the cause of this asymmetry.

For NGC 3114 \#80 and NGC 3532 \#413, we find at least two periods for each star on widely different timescales. With the given data set, we are unable to decide which period is due to classical rotation. In the phase diagrams of these two objects, the long-term trend was subtracted from the data. These two stars may be similar in some ways to HR 1297, a B9 Si star, for which periods of 1.06457 days and 15.749 days were reported by Adelman (2000). The astrophysical reason for this behaviour remains unknown but it is certainly connected to the highly variable properties of the stellar photosphere.

For NGC 3228 \#16 we used the period of 0.84 days, which fits the data more closely than the longer period of 0.94 days.

For NGC 2516 \#230, Warhurst (2004) presented I photometry and inferred a period of 1.943 days from a Fourier fit. This finding agrees with our result.

By combining the data for NGC 3114 \#234 with those published by North (1987), we obtain a more accurate measurement of the period of 2.31808 days. The shape of the Geneva photometry light curves shown in North (1987) are very similar to ours. When combining the data sets, we used a mean magnitude calculated from all seven filters as well as the individual ones.

\footnotetext{
3 http://www . peranso.com/
} 
E. Paunzen et al.: A photometric long-term study of chemically peculiar stars in open clusters

Table 1. Results for the observed sample of stars in eleven open clusters.

\begin{tabular}{|c|c|c|c|c|c|c|c|c|c|c|}
\hline Cluster & Name & $W$ & $\begin{array}{c}V \\
{[\mathrm{mag}]}\end{array}$ & $\begin{array}{c}\Delta a \\
{[\mathrm{mmag}]}\end{array}$ & Spec. & Mem & $\begin{array}{l}\Delta t \\
\text { [d] }\end{array}$ & Num & $\begin{array}{l}\text { Period } \\
\text { [d] }\end{array}$ & $\begin{array}{c}\text { Period }_{\text {Lit }} \\
{[\mathrm{d}]}\end{array}$ \\
\hline \multirow[t]{3}{*}{ Cr 132} & HD $56343^{a}$ & 27 & 9.25 & +35 & $\mathrm{CP}$ & - & 675.2737 & 15 & 3.25 & \\
\hline & HD 56046 & 10 & 7.59 & & B6 V & & & & & \\
\hline & HD 56162 & 12 & 7.80 & & A $1 \mathrm{~V}$ & & & & & \\
\hline \multirow[t]{6}{*}{ IC 4725} & BD-195044L & 98 & 10.20 & +16 & $\mathrm{CP}$ & $\mathrm{p}$ & 7.0674 & 5 & variable & \\
\hline & HD 170836 & 167 & 8.95 & +46 & $\mathrm{CP}$ & $\mathrm{y}$ & 1819.1000 & 10 & $0.9(?)$ & \\
\hline & HD 170860A & 153 & 9.40 & +22 & $\mathrm{CP}$ & $\mathrm{p}$ & 1819.1000 & 10 & variable & \\
\hline & HD 170719 & 91 & 8.10 & & B5 III & & & & & \\
\hline & HD 170820 & 150 & 7.38 & & K0 III & & & & & \\
\hline & HD 170682 & 50 & 7.95 & & B5 II/III & & & & & \\
\hline \multirow[t]{5}{*}{ NGC 2516} & CP-60944 & 112 & 9.85 & +21 & $\mathrm{CP}$ & $\mathrm{y}$ & 337.1385 & 17 & 1.10 & \\
\hline & HD 65712 & 230 & 9.86 & +70 & $\mathrm{CP}$ & $\mathrm{p}$ & 9.1297 & 13 & 1.88 & $1.943^{1}$ \\
\hline & HD 66295 & 26 & 9.10 & +45 & $\mathrm{CP}$ & $\mathrm{y}$ & 9.1303 & 11 & 2.45 & $2.45004^{2}$ \\
\hline & CP-60947 & 13 & 8.06 & & B8 V & & & & & \\
\hline & CP-60952 & 9 & 9.30 & & B9 V & & & & & \\
\hline \multirow[t]{7}{*}{ NGC 3114} & HD 87240 & 25 & 8.65 & +30 & $\mathrm{CP}$ & $\mathrm{p}$ & 1734.3087 & 28 & variable & \\
\hline & HD $87405^{a}$ & 108 & 8.50 & +17 & $\mathrm{CP}$ & $?$ & 1734.2957 & 28 & 2.113 & \\
\hline & HD 87752 & 211 & 9.82 & +6 & $\mathrm{CP}$ & $\mathrm{y}$ & 4.9909 & 5 & constant & \\
\hline & HD 304841 & 80 & 9.99 & +36 & $\mathrm{CP}$ & $\mathrm{p}$ & 349.1259 & 21 & $\begin{array}{r}0.6978 \\
\text { longer }^{b}\end{array}$ & \\
\hline & HD 304842 & 234 & 9.72 & +19 & $\mathrm{CP}$ & $\mathrm{y}$ & 1394.2079 & 15 & 2.31808 & $2.31873^{2}$ \\
\hline & HD 87137 & 8 & 8.30 & & B8 III & & & & & \\
\hline & HD 87349 & 86 & 8.62 & & B8 V & & & & & \\
\hline \multirow[t]{4}{*}{ NGC 3228} & HD $89856^{a}$ & 3 & 9.09 & +32 & $\mathrm{CP}$ & $\mathrm{n}$ & 1734.2579 & 28 & 4.556 & $4.565^{3}$ \\
\hline & HD 298053 & 16 & 10.69 & +15 & $\mathrm{CP}$ & $\mathrm{p}$ & 97.8085 & 21 & 0.84 or 0.94 & \\
\hline & HD 89900 & 5 & 8.19 & & A0 IV & & & & & \\
\hline & HD 89915 & 6 & 7.90 & & A $0 \mathrm{~V}$ & & & & & \\
\hline \multirow[t]{4}{*}{ NGC 3532} & HD 96040 & 413 & 10.00 & +60 & $\mathrm{CP}$ & $\mathrm{p}$ & 1392.1704 & 17 & $\begin{array}{c}1.13 \\
\text { longer }^{b}\end{array}$ & \\
\hline & HD 96729 & 449 & 10.00 & +65 & $\mathrm{CP}$ & $\mathrm{n}$ & 11.9983 & 12 & 1.39 & \\
\hline & HD 96011 & 467 & 9.05 & & $\mathrm{~A} 0 \mathrm{~V}$ & & & & & \\
\hline & HD 96226 & 409 & 8.03 & & B8 & & & & & \\
\hline \multirow[t]{4}{*}{ NGC 5460} & HD 122983 & 142 & 9.90 & +20 & $\mathrm{CP}$ & $\mathrm{p}$ & 1392.1247 & 17 & $3.4<\mathrm{P}<3.8$ & \\
\hline & HD 123225 & 55 & 8.85 & +14 & $\mathrm{CP}$ & $\mathrm{y}$ & 1385.1544 & 10 & constant & \\
\hline & HD 123202 & 79 & 8.90 & & B9 IV/V & & & & & \\
\hline & HD 123224 & 50 & 8.00 & & B8 II & & & & & \\
\hline \multirow[t]{5}{*}{ NGC 5662} & $\mathrm{CP}-566330$ & 85 & 10.89 & +33 & $\mathrm{CP}$ & $\mathrm{n}$ & 92.7977 & 17 & 0.81 & \\
\hline & HD $127924^{a}$ & 187 & 9.20 & +29 & $\mathrm{CP}$ & $\mathrm{p}$ & 97.8059 & 19 & 3.55 & \\
\hline & CP-56 6334 & 104 & 9.90 & & B8 V & & & & & \\
\hline & HD 127817 & 81 & 9.15 & & B7 IV & & & & & \\
\hline & HD 127835 & 97 & 9.40 & & B7 V & & & & & \\
\hline \multirow[t]{4}{*}{ NGC 6281} & HD 153947 & 9 & 8.80 & & $\mathrm{CP}$ & $\mathrm{n}$ & 1395.1398 & 16 & 2.6841 & $2.662^{4}$ \\
\hline & HD 153948 & 15 & 9.35 & +40 & $\mathrm{CP}$ & $\mathrm{y}$ & 1395.1359 & 16 & 1.64 & $1.6370^{2}$ \\
\hline & BD-37 11237 & 6 & 8.60 & & B9 & & & & & \\
\hline & BD-37 11241 & 5 & 8.30 & & A2 & & & & & \\
\hline \multirow[t]{5}{*}{ NGC 6405} & CD-32 13119 & 7 & 10.95 & +33 & $\mathrm{CP}$ & $\mathrm{p}$ & 6.0188 & 6 & data too poor & \\
\hline & HD 318100 & 19 & 8.82 & +67 & $\mathrm{CP}$ & $\mathrm{y}$ & 1819.0577 & 13 & data too poor & $1.03012^{2}$ \\
\hline & HD 318107 & 77 & 9.35 & +94 & $\mathrm{CP}$ & $\mathrm{y}$ & 1819.0681 & 13 & data too poor & $\begin{array}{c}4.527^{2} \\
9.7085^{5} \\
52.6^{2}\end{array}$ \\
\hline & HD 160260 & 70 & 8.35 & & B9 & & & & & \\
\hline & HD 160335 & 2 & 7.30 & & B3 V & & & & & \\
\hline \multirow[t]{3}{*}{ NGC 6475} & HD 162305 & 14 & 7.80 & +16 & $\mathrm{CP}$ & $\mathrm{y}$ & 1820.0393 & 14 & variable & \\
\hline & HD 320764 & 23 & 8.90 & +20 & $\mathrm{CP}$ & $\mathrm{y}$ & 1820.0480 & 14 & variable? & \\
\hline & HD 162515 & 42 & 6.52 & & B9 V & & & & & \\
\hline
\end{tabular}

Notes. The primary targets are denoted with a spectral class "CP". The data for the $V$ magnitude, the spectral classification of the comparison stars, and $\Delta a$ measurements were taken from WEBDA (number $W$ ); the membership flag as determined in Sect. 5. In addition, the results of our time series analysis with the time base of the observations $(\Delta t)$ and the number of data points (Num) for Strömgren $u v b y$ are listed. The derived periods are significant to the last listed digit.

${ }^{(a)}$ Hipparcos data available; results are discussed in Sect. 4; ${ }^{(b)}$ our data set shows also a longer period which can not be resolved.

(1) Warhurst (2004); ${ }^{(2)}$ North (1987); ${ }^{(3)}$ Koen \& Eyer (2002); ${ }^{(4)}$ North et al. (1988); ${ }^{(5)}$ Manfroid \& Mathys (2000). 
Table 2. Log of observations with the extinction coefficients for the Strömgren $u v b y$ filters.

\begin{tabular}{|c|c|c|c|c|c|c|}
\hline Teles. & Obs. & $\begin{array}{c}\text { Night } \\
\text { [JD] }\end{array}$ & $\begin{array}{c}k_{u} \\
{[\mathrm{mag}]}\end{array}$ & $\begin{array}{c}k_{v} \\
{[\mathrm{mag}]}\end{array}$ & $\begin{array}{c}k_{b} \\
{[\mathrm{mag}]}\end{array}$ & $\begin{array}{c}k_{y} \\
{[\mathrm{mag}]}\end{array}$ \\
\hline \multirow[t]{19}{*}{ Bochum } & \multirow[t]{19}{*}{ HMM } & 2446581 & 0.570 & 0.350 & 0.230 & 0.170 \\
\hline & & 2446582 & 0.570 & 0.350 & 0.230 & 0.170 \\
\hline & & 2446583 & 0.570 & 0.350 & 0.230 & 0.170 \\
\hline & & 2446584 & 0.570 & 0.350 & 0.230 & 0.170 \\
\hline & & 2446585 & 0.570 & 0.350 & 0.230 & 0.170 \\
\hline & & 2446587 & 0.570 & 0.350 & 0.230 & 0.170 \\
\hline & & 2447963 & 0.570 & 0.350 & 0.230 & 0.170 \\
\hline & & 2447964 & 0.570 & 0.350 & 0.230 & 0.170 \\
\hline & & 2447965 & 0.570 & 0.350 & 0.230 & 0.170 \\
\hline & & 2447966 & 0.570 & 0.350 & 0.230 & 0.170 \\
\hline & & 2447967 & 0.570 & 0.350 & 0.230 & 0.170 \\
\hline & & 2447968 & 0.570 & 0.350 & 0.230 & 0.170 \\
\hline & & 2447969 & 0.570 & 0.350 & 0.230 & 0.170 \\
\hline & & 2447970 & 0.570 & 0.350 & 0.230 & 0.170 \\
\hline & & 2447971 & 0.570 & 0.350 & 0.230 & 0.170 \\
\hline & & 2447972 & 0.570 & 0.350 & 0.230 & 0.170 \\
\hline & & 2447973 & 0.570 & 0.350 & 0.230 & 0.170 \\
\hline & & 2447974 & 0.570 & 0.350 & 0.230 & 0.170 \\
\hline & & 2447976 & 0.570 & 0.350 & 0.230 & 0.170 \\
\hline \multirow[t]{10}{*}{ SAT } & \multirow[t]{10}{*}{ CT } & 2448307 & 0.570 & 0.350 & 0.230 & 0.170 \\
\hline & & 2448308 & 0.570 & 0.350 & 0.230 & 0.170 \\
\hline & & 2448309 & 0.570 & 0.350 & 0.230 & 0.170 \\
\hline & & 2448310 & 0.570 & 0.350 & 0.230 & 0.170 \\
\hline & & 2448311 & 0.570 & 0.350 & 0.230 & 0.170 \\
\hline & & 2448313 & 0.570 & 0.350 & 0.230 & 0.170 \\
\hline & & 2448314 & 0.570 & 0.350 & 0.230 & 0.170 \\
\hline & & 2448315 & 0.570 & 0.350 & 0.230 & 0.170 \\
\hline & & 2448308 & 0.570 & 0.350 & 0.230 & 0.170 \\
\hline & & 2448308 & 0.570 & 0.350 & 0.230 & 0.170 \\
\hline \multirow[t]{15}{*}{ SAT } & \multirow{15}{*}{$\mathrm{HH}$} & 2448392 & 0.570 & 0.350 & 0.230 & 0.170 \\
\hline & & 2448393 & 0.570 & 0.350 & 0.230 & 0.170 \\
\hline & & 2448394 & 0.570 & 0.350 & 0.230 & 0.170 \\
\hline & & 2448398 & 0.570 & 0.350 & 0.230 & 0.170 \\
\hline & & 2448400 & 0.570 & 0.350 & 0.230 & 0.170 \\
\hline & & 2448405 & 0.570 & 0.350 & 0.230 & 0.170 \\
\hline & & 2448407 & 0.592 & 0.369 & 0.244 & 0.184 \\
\hline & & 2448408 & 0.592 & 0.369 & 0.244 & 0.184 \\
\hline & & 2448409 & 0.592 & 0.369 & 0.244 & 0.184 \\
\hline & & 2448410 & 0.592 & 0.369 & 0.244 & 0.184 \\
\hline & & 2448411 & 0.735 & 0.455 & 0.320 & 0.240 \\
\hline & & 2448413 & 0.575 & 0.355 & 0.230 & 0.170 \\
\hline & & 2448414 & 0.575 & 0.355 & 0.230 & 0.170 \\
\hline & & 2448415 & 0.575 & 0.355 & 0.230 & 0.170 \\
\hline & & 2448416 & 0.575 & 0.355 & 0.230 & 0.170 \\
\hline \multirow[t]{7}{*}{ SAT } & \multirow[t]{7}{*}{ HMM } & 2448638 & 0.720 & 0.480 & 0.350 & 0.270 \\
\hline & & 2448639 & 0.660 & 0.420 & 0.305 & 0.240 \\
\hline & & 2448640 & 0.630 & 0.380 & 0.280 & 0.230 \\
\hline & & 2448641 & 0.590 & 0.360 & 0.250 & 0.200 \\
\hline & & 2448642 & 0.600 & 0.355 & 0.250 & 0.190 \\
\hline & & 2448643 & 0.600 & 0.360 & 0.255 & 0.205 \\
\hline & & 2448644 & 0.590 & 0.350 & 0.240 & 0.190 \\
\hline
\end{tabular}

Notes. For the majority of the observations, the standard extinction coefficients for LaSilla fit the data very well.

The deduced period for these different procedures is numerically the same up to the fifth digit. Our light curves do not show as prominent a secondary minimum as those from the Geneva data. However, we are unable to definitely rule out a double wave signature, especially for Strömgren $y$.

For the three CP stars in NGC 6405, the data are of too poor quality to draw any definitive conclusion. Even the combination with previously published photometry does not result in any significant improvement of the situation. However, since our photometric data will be publicly available, they are an important contribution to long-term studies of this star group.

Four stars of our sample, namely IC 4725 \#98, IC 4725 \#153, NGC 3114 \#25, NGC $6475 \# 14$, and NGC 6475 \#23, are certainly variable according to the detection limits of the applied time-series methods, but we are unable to deduce reliable periods from our data sets. These objects are worthwhile targets for follow-up observations. The time series analysis of our data set for NGC 6475 \#23 is rather inconclusive. From the different results and an inspection of the light curve by eye, we conclude that this star is indeed variable.

In Fig. 2, the phase diagrams of all stars except for IC 4725 \#98, which has few data points, are plotted. For each object we took the period derived for the differential Strömgren $v$ magnitudes. The range of calculated periods agrees well with those of the other program stars.

\section{Hipparcos photometry}

For four target stars, we retrieved Hipparcos data and analysed them together with the ground-based data, i.e. Cr 132 \#27 (HIC 120047), NGC 3114 \#108 (HIC 49218), NGC 3228 \#3 (HIC 50677) and NGC 5662 \#187 (HIC 71398). Among these stars, only NGC 3228 \#3 was flagged as variable from the Hipparcos data.

The data sets cover a typical time frame of three years with about 130 measurements of each star.

Following the advice given in the catalogue publication, measurements derived from data with identified reduction problems were not considered. Since the Hipparcos data have uncertainties of between $1 \%$ and $2 \%$ at the involved magnitudes, comparable to the expected amplitudes of the light variation, a weighted average of data obtained over a small section of the same rotation cycle, typically up to $0.05 P_{\text {rot }}$, was used. Indeed, a smaller amount of more accurate observations is more helpful to period searches and Hipparcos observations tend to be clustered in time because of the observing procedure (two observations within $20 \mathrm{~min}$, repeated commonly two hours later).

The time series analysis was performed such that we attempted to identify the optimal period by fitting to all quantities $m$ (all individual Strömgren filters and $H_{p}$ ) a function

$m=m_{0}+A\left(\cos 2 \pi\left(\phi-\phi_{0}\right)+B \cos 4 \pi\left(\phi-\phi_{1}\right)\right)$,

where $m_{0}, A, B, \phi_{0}$ and $\phi_{1}$ are free parameters. When the shape of the light curves did not significantly differ in the filters, we took the same values of $B, \phi_{0}$, and $\phi_{1}$ in all channels, only varying $m_{0}$ and $A$ from filter to filter.

Cr 132 \#27: this is the faintest Hipparcos target of our sample. The analysis of the Hipparcos data alone results in a period that is twice as long as deduced from the uvby photometry. This may be indicative of an apparent double wave. However, merging the two data sets does not help us to prove with this interpretation.

NGC 3114 \#108: from the uvby photometry we derive a period of 2.113 days. Adding the Hipparcos measurements, the range of possible periods can be narrowed down to 2.1250-2.1265 days. This interval contains two solutions with overlapping significance intervals at the $1.5 \sigma$ level. The highest probability occurs at 2.1253 and at 2.1262 days, respectively.

$N G C$ 3228 \#3: the uvby light curves deduced for this object are rather noisy, but the derived period is consistent with that derived from the Hipparcos observations and is compatible with 
E. Paunzen et al.: A photometric long-term study of chemically peculiar stars in open clusters
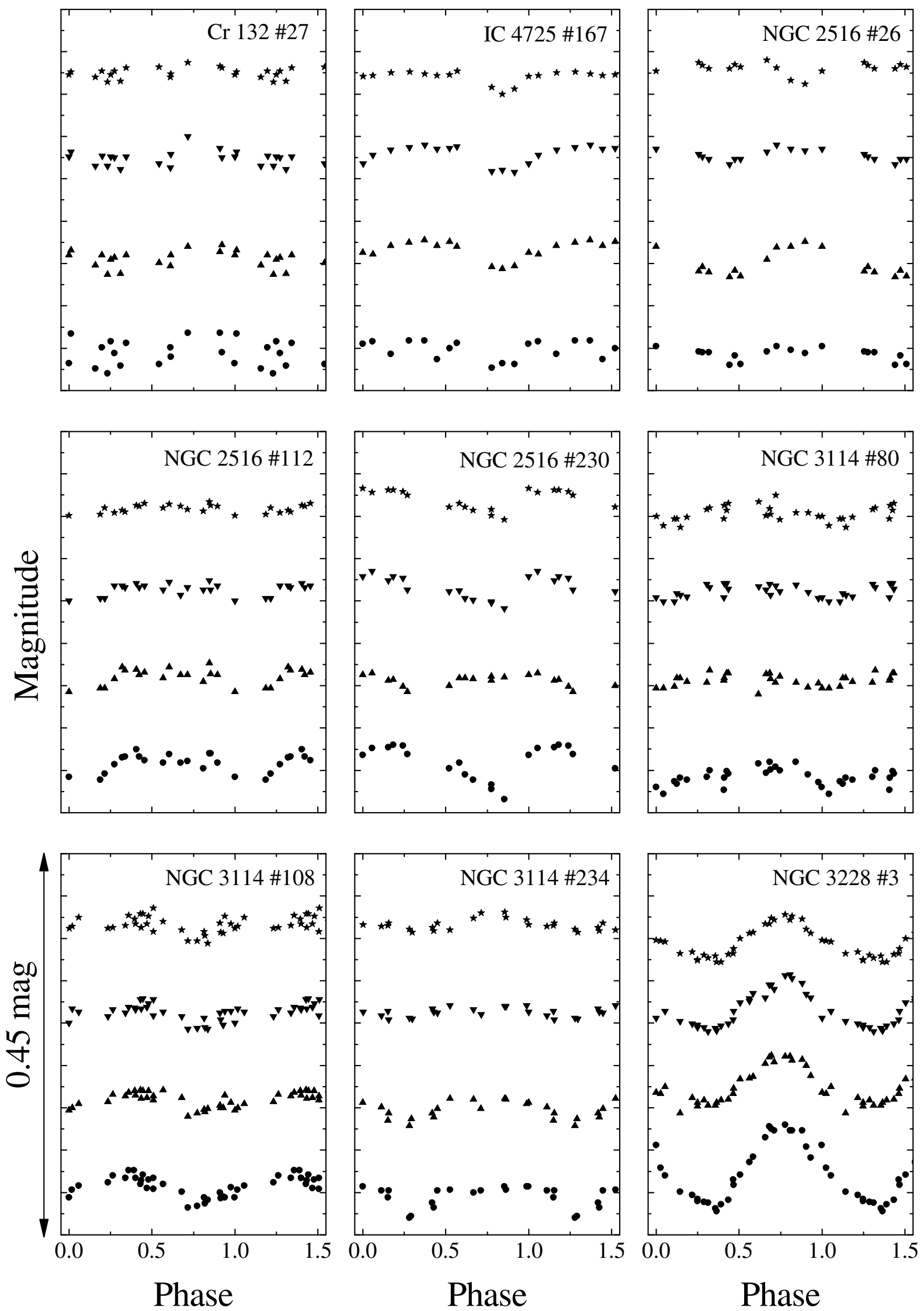

Fig. 1. Phase diagrams of differential Strömgren $u$ (circles), $v$ (upper triangles), $b$ (lower triangles), and $y$ (star symbols) magnitudes for the variable program stars with well-established periods. The interval between two major ticks is 0.05 mag. 

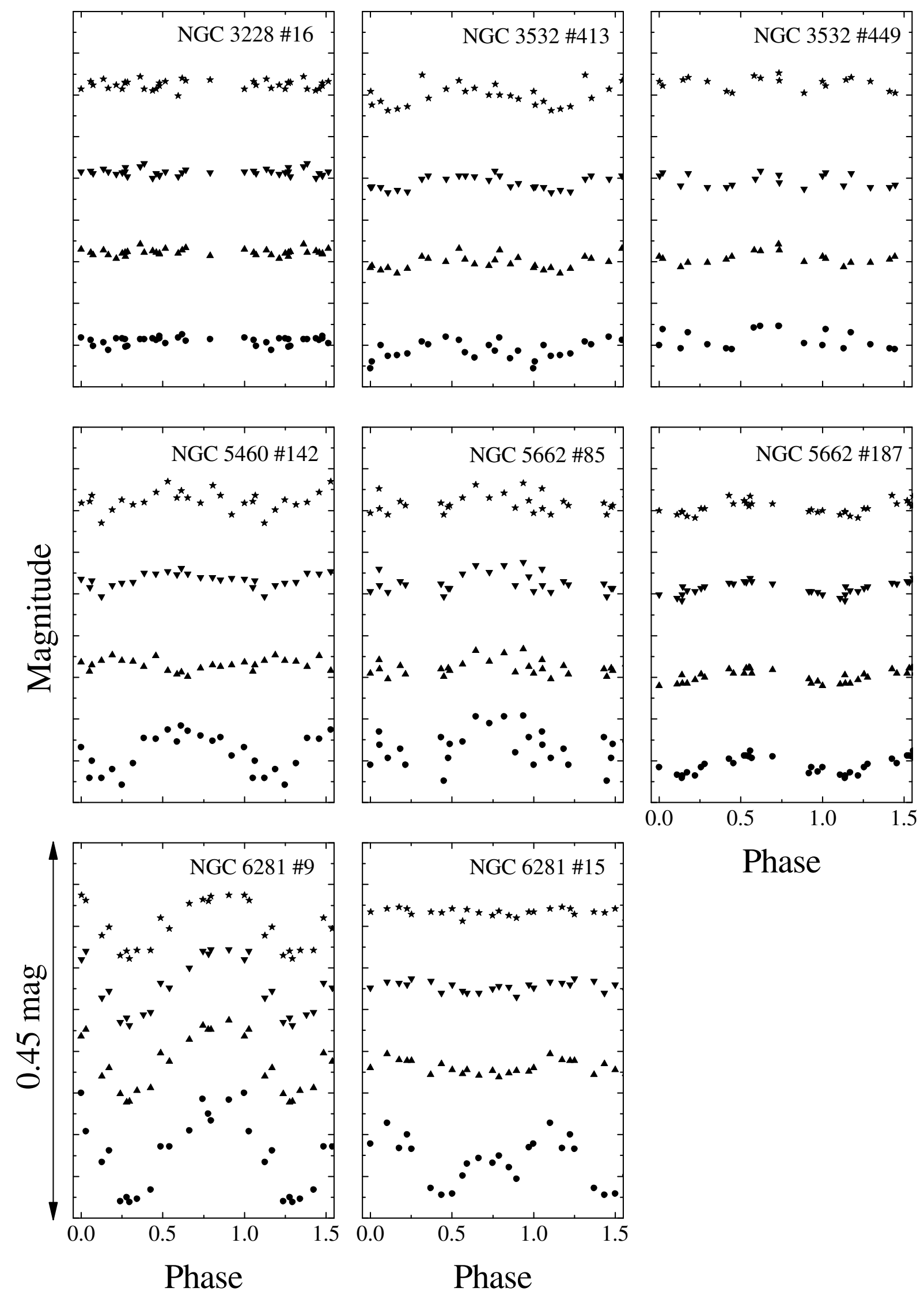

Phase

Fig. 1. continued. 

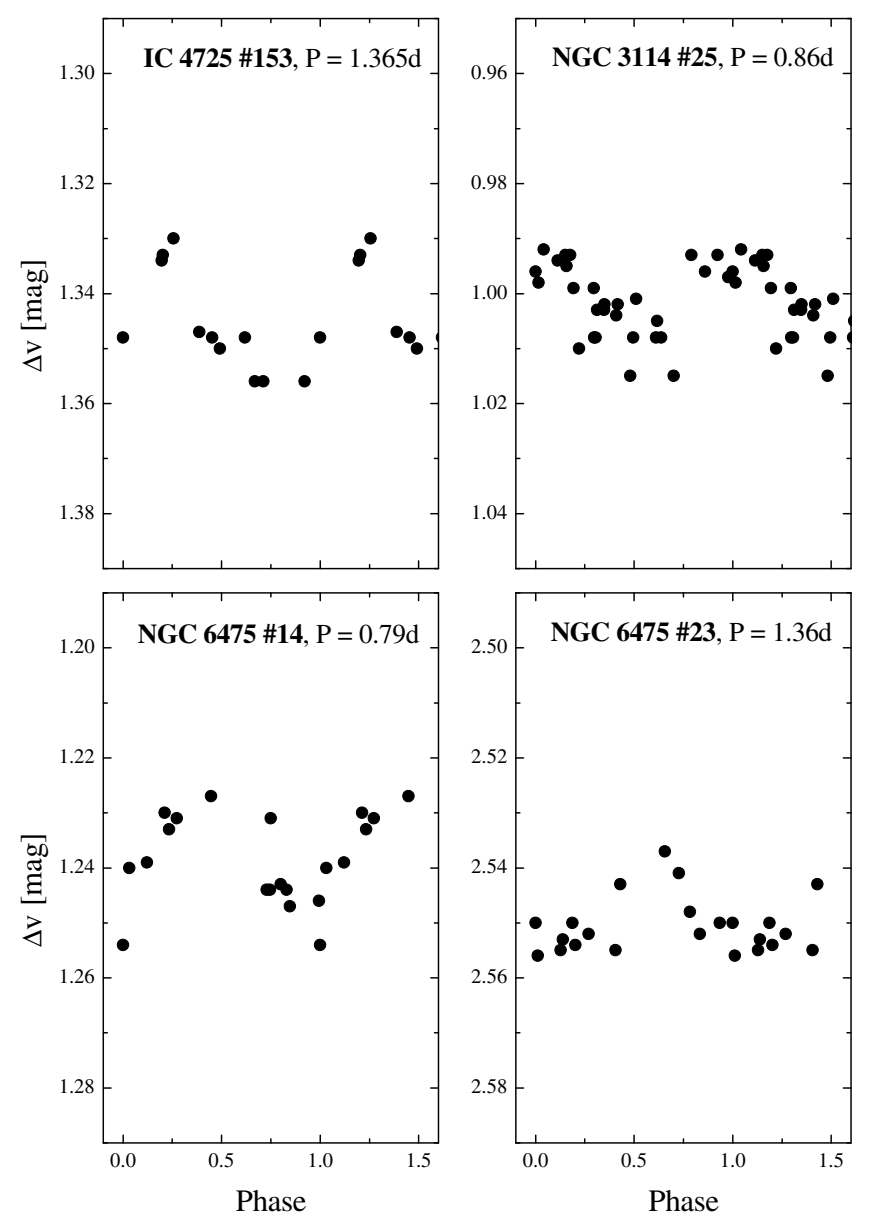

Fig. 2. Phase diagrams of differential Strömgren $v$ magnitudes for the variable program stars with an uncertain period.

the result obtained by Koen \& Eyer (2002) from the Hipparcos data.

NGC 5662 \#187: in spite of the low amplitude, the periodicity is still detectable from an analysis of the Hipparcos magnitudes. It is most clearly visualized by forming weighted averages over individual measurements that were obtained within one fifteenth of the same stellar rotation cycle. The combination of both uvby and Hipparcos photometry allows us to deduce the period given in Table 1 .

\section{Membership}

In more in-depth investigations (e.g., of evolutionary aspects), it is essential to know whether the respective object belongs to the cluster. Several criteria can be used to determine membership. Apart from photometric criteria, the most commonly used are certainly based on proper motion. These, and both radial velocities and parallaxes would help assign membership, but data of the latter two in particular are unfortunately sparsely available for our programme objects. Several comprehensive studies are available, which already provide membership probabilities in open clusters using proper motion (Baumgardt et al. 2000; Dias et al. 2001, 2006; Kharchenko et al. 2004), from which we compiled their results as well as their respective data source. Furthermore, we considered the results of Javakhishvili et al. (2006), which are based on the same data (Tycho-2) as Dias et al. (2001), but a different method was applied to obtain membership probabilities. These datasets were complemented with data from other previous studies (Constantine et al. 1969; Francic 1989; King 1978a,b, 1980) for NGC 6475, IC 4725, NGC 3532, NGC 2516, and NGC 5662, respectively.

Since slightly different cluster means were determined in the various studies and also different scales were used, the use of probability values of a single source can lead to wrong conclusions. They have to be compared with other investigations, including those examining the respective source data and the deviation from the cluster mean motion. A demonstrative example are the results for HD 127924, for which Baumgardt et al. (2000) list $9 \%$, although this star deviates 2.6 mas only from the cluster mean. The same value was obtained by Dias et al. (2006) with a deviation of 7.8 mas, but their cluster mean differs significantly from all other studies.

We therefore followed the approach given in Landstreet et al. (2007) to divide membership into y (member), p (probable member), ? (probably non-member), and n (non-member). We refer to Landstreet et al. (2007) for a more detailed explanation of the problems connected with membership probabilities. We queried the latest release of UCAC3 (Zacharias et al. 2009) to check the consistency of the proper motions and assigned a membership flag to the programme objects based on this property. As in Landstreet et al. (2007), we performed a similar assignment for the position of the objects in the Hertzsprung-Russell diagram (HRD) relative to the isochrone of the respective cluster age. Furthermore, parallaxes were extracted from the new Hipparcos reduction by van Leeuwen (2007) and compared with the cluster distances. As a fourth membership parameter, we used radial velocities taken from Amieux \& Burnage (1981), Francic (1989), Gieseking (1985), González \& Lapasset (2000), González \& Lapasset (2001), Landstreet et al. (2008), as well as Luck et al. (2000), which were compared with both the cluster mean of the respective sources and those of Mermilliod et al. (2008). However, these results have to be evaluated with caution, if only a few measurements are available since binarity cannot be excluded. Using all available information, a final membership flag was assigned, which is given in Table 4 together with the result by Landstreet et al. (2007). In the following, non-members, with results that differ significantly from both Landstreet et al. (2007) and other results are discussed.

Three stars (HD 89856, 96729, and 153947) that deviate strongly in the HRD: only unusually large errors in temperature of the order of about $2000 \mathrm{~K}$ would put them close to the corresponding isochrone. All other available membership parameters are also uncertain, so they were classified as non-members in agreement with Landstreet et al. (2007). Furthermore, the position of HD 153947, not included in this reference, is located about four times the cluster radius (4', Dias et al. 2002) away from the cluster center.

HD 65712 in NGC 2516 is also located far from the cluster center, at a distance of about three times the cluster radius of $15^{\prime}$ (Dias et al. 2002). Since the two available parameters are indicative of membership, and the star is just within the much larger radius determined by Kharchenko et al. (2004), a probable membership was deduced.

For the star HD 87240 in NGC 3114, its radial velocity data and the position in the HRD support membership, in contrast to most proper motions. However, the data of UCAC3, which deviates strongly from all others, do not contradict membership. Therefore, a probable membership was concluded for this star. Several membership criteria are available for HD 87405 in the same cluster. The radial velocities of González \& Lapasset (2001) exclude membership, listing a probability of $0 \%$. In contrast, the results by Amieux \& Burnage (1981) are in good 
Table 3. The observed open clusters with their parameters based on the list by Paunzen \& Netopil (2006).

\begin{tabular}{lllcr}
\hline \hline Cluster & Name & $\begin{array}{c}D \\
{[\mathrm{pc}]}\end{array}$ & $\begin{array}{c}E(B-V) \\
{[\mathrm{mag}]}\end{array}$ & $\begin{array}{c}\text { Age } \\
{[\mathrm{Myr}]}\end{array}$ \\
\hline Cr 132 & C0712-310 & $438(39)$ & $0.03(1)$ & $23(10)$ \\
IC 4725 & C1828-192 & $615(65)$ & $0.48(2)$ & $75(15)$ \\
NGC 2516 & C0757-607 & $402(32)$ & $0.10(4)$ & $141(21)$ \\
NGC 3114 & C1001-598 & $914(19)$ & $0.06(3)$ & $128(38)$ \\
NGC 3228 & C1019-514 & $511(22)$ & $0.02(1)$ & $102(17)$ \\
NGC 3532 & C1104-584 & $492(8)$ & $0.04(1)$ & $262(46)$ \\
NGC 5460 & C1404-480 & $673(76)$ & $0.12(2)$ & $167(54)$ \\
NGC 5662 & C1431-563 & $684(60)$ & $0.32(1)$ & $77(20)$ \\
NGC 6281 & C1701-378 & $516(35)$ & $0.15(1)$ & $285(58)$ \\
NGC 6405 & C1736-321 & $473(16)$ & $0.14(2)$ & $71(21)$ \\
NGC 6475 & C1750-348 & $258(21)$ & $0.07(3)$ & $267(62)$ \\
\hline
\end{tabular}

Table 4. Membership of the programme objects.

\begin{tabular}{|c|c|c|c|}
\hline \multirow[t]{2}{*}{ Cluster } & \multirow[t]{2}{*}{ CP Star } & \multicolumn{2}{|c|}{ Membership } \\
\hline & & $\mu / v / \mathrm{ph} / \pi$ & final \\
\hline Cr 132 & HD 56343 & $\mathrm{y} /-/ \mathrm{y} / \mathrm{n}$ & $-(\mathrm{p})^{a}$ \\
\hline \multirow[t]{3}{*}{ IC 4725} & BD-19 5044L & $\mathrm{p} /-/ \mathrm{p} /-$ & $\mathrm{p}(\mathrm{p})$ \\
\hline & HD 170836 & $\mathrm{y} / \mathrm{y} / \mathrm{p} /-$ & $y(p)$ \\
\hline & HD 170860 & $\mathrm{p} / \mathrm{n}^{b} / \mathrm{y} /-$ & $\mathrm{p}(\mathrm{p})$ \\
\hline \multirow[t]{3}{*}{ NGC 2516} & CP-60 944 & $\mathrm{y} /-/ \mathrm{y} /-$ & $\mathrm{y}(\mathrm{y})^{a}$ \\
\hline & HD 65712 & $\mathrm{y} /-/ \mathrm{y} /-$ & $\mathrm{p}(\mathrm{y})^{a}$ \\
\hline & HD 66295 & y/y/y/- & y (y) \\
\hline \multirow{5}{*}{ NGC 3114} & HD 87240 & $\mathrm{p} / \mathrm{y} / \mathrm{y} /-$ & $\mathrm{p}(\mathrm{n})^{a}$ \\
\hline & HD 87405 & $\mathrm{p} / \mathrm{n} / \mathrm{y} / \mathrm{p}$ & $?(?)^{a}$ \\
\hline & HD 87752 & $\mathrm{p} / \mathrm{y} / \mathrm{y} /-$ & $\mathrm{y}(-)$ \\
\hline & HD 304841 & $\mathrm{p} /-/ \mathrm{y} /-$ & $\mathrm{p}(\mathrm{p})$ \\
\hline & HD 304842 & $\mathrm{p} / \mathrm{y} / \mathrm{y} /-$ & $y(p)$ \\
\hline \multirow{2}{*}{ NGC 3228} & HD 89856 & $\mathrm{p} /-/ \mathrm{n} / \mathrm{p}$ & $\mathrm{n}(\mathrm{n})^{a}$ \\
\hline & HD 298053 & $\mathrm{p} /-/ \mathrm{y} /-$ & $\mathrm{p}(\mathrm{p})$ \\
\hline \multirow[t]{2}{*}{ NGC 3532} & HD 96040 & $\mathrm{p} /-/ \mathrm{p} /-$ & $\mathrm{p}(\mathrm{p})$ \\
\hline & HD 96729 & $\mathrm{p} /-/ \mathrm{n} /-$ & $\mathrm{n}(?)^{a}$ \\
\hline \multirow[t]{2}{*}{ NGC 5460} & HD 122983 & $\mathrm{y} /-/ \mathrm{p} /-$ & $\mathrm{p}(\mathrm{p})$ \\
\hline & HD 123225 & $\mathrm{y} /-/ \mathrm{y} /-$ & y (y) \\
\hline \multirow[t]{2}{*}{ NGC 5662} & CP-56 6330 & $\mathrm{n} /-/ \mathrm{y} /-$ & $\mathrm{n}(\mathrm{n})^{a}$ \\
\hline & HD 127924 & $\mathrm{y} /-/ \mathrm{p} / \mathrm{p}$ & $\mathrm{p}(\mathrm{y})$ \\
\hline \multirow[t]{2}{*}{ NGC 6281} & HD 153947 & $\mathrm{p} /-/ \mathrm{n} /-$ & $\mathrm{n}(-)^{a}$ \\
\hline & HD 153948 & $\mathrm{y} / \mathrm{p} / \mathrm{y} /-$ & y (y) \\
\hline \multirow[t]{3}{*}{ NGC 6405} & CD-32 13119 & $\mathrm{p} /-/ \mathrm{y} /-$ & $\mathrm{p}(\mathrm{p})$ \\
\hline & HD 318100 & y/y/y/- & y (y) \\
\hline & HD 318107 & $\mathrm{y} /-/ \mathrm{y} /-$ & $\mathrm{y}(\mathrm{y})$ \\
\hline \multirow[t]{2}{*}{ NGC 6475} & HD 162305 & $\mathrm{y} / \mathrm{y} / \mathrm{y} /-$ & $\mathrm{y}(\mathrm{y})$ \\
\hline & HD 320764 & $\mathrm{y} / \mathrm{y} / \mathrm{y} /-$ & $\mathrm{y}(\mathrm{p})$ \\
\hline
\end{tabular}

Notes. The final membership was deduced from individual ones based on proper motion $(\mu)$, radial velocity $(v)$, position in the HRD (ph), and parallaxes $(\pi)$. In parenthesis, the membership obtained by Landstreet et al. (2007) is given.

(a) membership discussed in the text; ${ }^{(b)}$ only a single measurement by Landstreet et al. (2008); binarity cannot be excluded.

agreement with their measurement of cluster mean. Both presented several measurements that provided no evidence of binarity. We prefer the results of the first of these aforementioned studies, since they are based on higher resolution CCD measurements than the photographic plates used by Amieux \& Burnage (1981). This star was therefore classified as a probable nonmember as in Landstreet et al. (2007).

According to its proper motion and all available studies, the star CP-56 6330 cannot be a member of the cluster NGC 5662, although its position in the HRD suggests the contrary. The final classification as a non-member agrees with Landstreet et al. (2007). Its proper motion as well as its HRD position fully
Table 5. Derived logarithmic luminosities and effective temperatures of our programme stars.

\begin{tabular}{lcc}
\hline \hline CP Star & $\log T_{\text {eff }}$ & $\log L / L_{\odot}$ \\
\hline BD -19 5044L & 4.094 & 2.25 \\
CD -32 13119 & 3.914 & 1.05 \\
CP -56 6330 & 4.088 & 1.95 \\
CP -60 944 & 4.095 & 2.24 \\
HD 56343 & 4.063 & 1.70 \\
HD 65712 & 4.000 & 1.52 \\
HD 66295 & 4.043 & 1.75 \\
HD 87240 & 4.092 & 2.29 \\
HD 87405 & 4.092 & 2.74 \\
HD 87752 & 4.105 & 2.28 \\
HD 89856 & 4.165 & 2.16 \\
HD 96040 & 4.026 & 1.42 \\
HD 96729 & 4.083 & 1.57 \\
HD 122983 & 4.032 & 1.89 \\
HD 123225 & 4.084 & 2.40 \\
HD 127924 & 4.113 & 2.50 \\
HD 153947 & 4.116 & 2.29 \\
HD 153948 & 4.026 & 1.84 \\
HD 162305 & 4.008 & 1.74 \\
HD 170836 & 4.162 & 2.89 \\
HD 170860 A & 4.137 & 2.63 \\
HD 298053 & 3.936 & 1.09 \\
HD 304841 & 4.089 & 2.15 \\
HD 304842 & 4.094 & 2.25 \\
HD 318100 & 4.025 & 1.60 \\
HD 318107 & 4.070 & 1.90 \\
HD 320764 & 3.932 & 1.26 \\
\hline
\end{tabular}

support membership of HD 56343 to Collinder 132, but its parallax $(0.26 \pm 1.1)$ would place it as a background object, which also holds for the "original" Hipparcos data $(0.66 \pm 1.09)$. However, we note that the nature of this open cluster continues to be discussed in the literature (Clariá 1977; Baumgardt 1998; Caballero \& Dinis 2008). Therefore, we are not able to draw a final membership conclusion for this star. For the visual binary CP-60 944, both components are at least probable members, whereas the A component is according to its proper motion and position in the HRD a definite member (see also Landstreet et al. 2007).

\section{Results and conclusions}

We have investigated photometrically a total of $27 \mathrm{CP}$ stars within the boundaries of open clusters.

We established variability for fourteen CP stars with previously unknown rotation periods, and confirmed the rotation periods of eight more stars with an increase in precision for two of them.

The applied open cluster parameters (Table 3) are averaged ones based on a set of literature values. Starting with the comprehensive compilation by Paunzen \& Netopil (2006), we searched the literature for additional (new or overseen) parameters. They were all checked for plausibility by using appropriate isochrones and available photometric data taken from the WEBDA database. Significant different results were removed and finally a strict average and standard deviations were calculated.

The $\log T_{\text {eff }}$ values were calculated using the calibration of Netopil et al. (2008) for CP stars. We made use of the 
E. Paunzen et al.: A photometric long-term study of chemically peculiar stars in open clusters

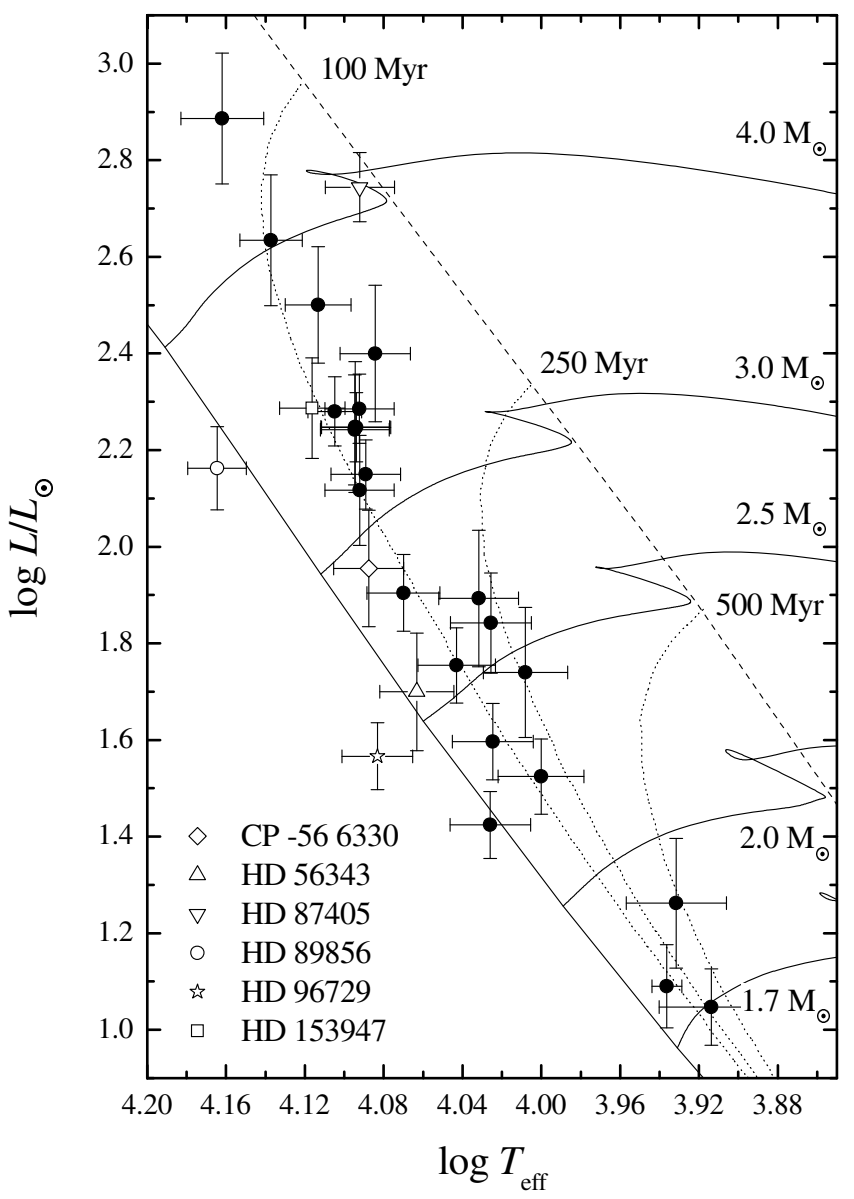

Fig. 3. The location of the investigated $\mathrm{CP}$ stars in a $\log L / L_{\odot}$ versus $\log T_{\text {eff }}$ diagram. Filled symbols denote members, whereas open symbols are non-members. The dashed line denotes the terminal-age mainsequence; the evolutionary tracks for individual masses are interpolated within the solar metallicity ones listed by Schaller et al. (1992), Schaerer et al. (1993a), Schaerer et al. (1993b), and Charbonnel et al. (1993).

GCPD catalogue ${ }^{4}$ and the WEBDA database to extract all available photometric data of the programme stars. To obtain absolute magnitudes (assuming that all programme stars are indeed cluster members), we took averaged values and the mean cluster distances (see Table 3). Since the stars in NGC 2516 in particular, exhibit a strong differential reddening, individual reddening values were determined whenever possible for all objects as suggested by Netopil et al. (2008). Within the latter reference a bolometric correction for magnetic CP stars was also introduced, which was used to calculate the luminosity. For the remaining Am and HgMn objects, the bolometric correction by Balona (1994) for normal stars was applied. In Table 5, the individual values are listed.

Figure 3 shows the location of the investigated CP stars in a $\log L / L_{\odot}$ versus $\log T_{\text {eff }}$ diagram. The dashed line denotes the terminal-age main-sequence. The evolutionary tracks for individual masses and ages are interpolated between the solar metallicity ones listed by Schaller et al. (1992), Schaerer et al. (1993a), Schaerer et al. (1993b), and Charbonnel et al. (1993).

Two stars, HD 89856 and HD 96729 are located below the ZAMS and deviate significantly from the apparent cluster age (Table 3). These objects are definite non-members according to our analysis and that of Landstreet et al. (2007). We also marked

\footnotetext{
${ }^{4}$ http://obswww. unige.ch/gcpd/gcpd.html
}

the location of the other questionable cluster members as discussed in Sect. 5.

The target stars cover the typical mass range for mid B to late A type main-sequence objects from about $1.7 M_{\odot}$ to $4.5 M_{\odot}$ as other members of this group (Pöhnl et al. 2005). The photometric periods of $0.7-4.5$ days are consistent with the typical rotation velocities of CP stars (North et al. 1992).

There is a hint of the period decreasing with increasing age, in particular for stars with a mass below $3 M_{\odot}$, but this is not statistically significant due to poor number statistics. The same is true for a possible correlation of the period with the stellar mass and effective temperature. However, with the ongoing observations, more light will be shed on these important topics.

Our observations fill an important gap in previous photometric long-time studies of CP stars. The apparent open cluster members are excellent targets for follow-up observations, based on for example polarimetry, high-resolution spectroscopy, and surface mapping techniques.

Follow-up observations within our framework are already under way with the Rapid Eye Mount (REM) telescope at La Silla. This will help us to understand the apparent stellar cycles, such as that of the Sun, for this group of magnetic peculiar objects.

Acknowledgements. We would like to thank Gautier Mathys for significantly improving this paper. This work was supported by the financial contributions of the Austrian Agency for International Cooperation in Education and Research (WTZ CZ-11/2008, CZ-10/2010, and HR-14/2010), the City of Vienna (Hochschuljubiläumsstiftung project: H-1930/2008), the Forschungsstipendium der Universität Wien (F-416), a MOEL grant of the ÖFG (Project \#388) and a travel grant from the Swedish Research Council. U.H. acknowledges support from the Swedish National Space Board. This research has made use of the WEBDA database, operated at the Institute for Astronomy of the University of Vienna.

\section{References}

Adelman, S. J. 2000, A\&AS, 146, 13

Amieux, G., \& Burnage, R. 1981, A\&AS, 44, 101

Babcock, H. W. 1947, ApJ, 105, 105

Balona, L. A. 1994, MNRAS, 268, 119

Baumgardt, H. 1998, A\&A, 340, 402

Baumgardt, H., Dettbarn, C., \& Wielen, R. 2000, A\&AS, 146, 251

Caballero, J. A., \& Dinis, L. 2008, Astron. Nachr., 329, 801

Charbonnel, C., Meynet, G., Maeder, A., Schaller, G., \& Schaerer, D. 1993, A\&AS, 101, 415

Clariá, J. J. 1977, PASP, 89, 803

Constantine, S. M., Harris, B. J., \& Nikoloff, I. 1969, Proc. Astron. Soc. Aust., 1,207

Deeming T. J. 1975, Ap\&SS, 36, 137

Dias, W. S., Lépine, J. R. D., \& Alessi, B. S. 2001, A\&A, 376, 441

Dias, W. S., Alessi, B. S., Moitinho, A., \& Lépine, J. R. D. 2002, A\&A, 389, 871

Dias, W. S., Assafin, M., Flório, V., Alessi, B. S., \& Líbero, V. 2006, A\&A, 446, 949

Francic, S. P. 1989, AJ, 98, 888

Gieseking, F. 1985, A\&AS, 61, 75

González, J. F., \& Lapasset, E. 2000, AJ, 119, 2296

González, J. F., \& Lapasset, E. 2001, AJ, 121, 2657

Guthnik, P., \& Prager, R. 1914, Veröffentl. der königl. Sternwarte BerlinBabelsberg, 1

Hensberge, H., de Loore, C., Zuiderwijk, E. J., \& Hammerschlag-Hensberge, G. 1977, A\&A, 54, 443

Javakhishvili, G., Kukhianidze, V., Todua, M., \& Inasaridze, R. 2006, A\&A, 447, 915

Kharchenko, N. V., Piskunov, A. E., Röser, S., Schilbach, E., \& Scholz, R.-D. 2004, Astron. Nachr., 325, 740

King, D. S. 1978a, J. Proc. Roy. Soc. New South Wales, 111, 1

King, D. S. 1978b, J. Proc. Roy. Soc. New South Wales, 111, 61

King, D. S. 1980, J. Proc. Roy. Soc. New South Wales, 113, 7

Koen, C., \& Eyer, L. 2002, MNRAS, 331, 45

Lafler, J., \& Kinman, T. D. 1976, ApJS, 11, 216

Landstreet, J. D., Bagnulo, S., Andretta, V., et al. 2007, A\&A, 470, 685

Landstreet, J. D., Silaj, J., Andretta, V., et al. 2008, A\&A, 481, 465 
Lehmann, H., Tkachenko, A., Fraga, L., Tsymbal, V., \& Mkrtichian, D. E. 2007, A\&A, 471, 941

Luck, R. E., Andrievsky, S. M., Kovtyukh, V. V., Korotin, S. A., \& Beletsky, Yu. V. 2000, A\&A, 361, 189

Maitzen, H. M. 1976, A\&A, 51, 223

Maitzen, H. M. 1980, A\&A, 89, 230

Maitzen, H. M. 1993, A\&AS, 102, 1

Maitzen, H. M., \& Hensberge, H. 1981, A\&A, 96, 151

Maitzen, H. M., Weiss, W. W., \& Wood, H. J. 1980, A\&A, 81, 323

Manfroid, J., \& Mathys, G. 2000, A\&A, 364, 689

Mathys, G., Maitzen, H. M., North, P., et al. 1989, The Messenger, 55, 41

Mathys, G., Hubrig, S., Landstreet, J. D., Lanz, T., \& Manfroid, J. 1997, A\&AS, 123,353

Mermilliod, J.-C., Mayor, M. \& Udry, S. 2008, A\&A, 485, 303

Mikulášek, Z., Janik, J., Zverko, J., et al. 2007, Astron. Nachr., 328, 10

Mikulášek, Z., Krtička, J., Henry, G. W., et al. 2010, A\&A, 511, L7

Netopil, M., Paunzen, E., Maitzen, H. M., North, P., \& Hubrig, S. 2008, A\&A, 491,545
North, P. 1987, A\&AS, 70, 247

North, P., Jasniewicz, G., \& Waelkens, Ch. 1988, IBVS, 3199

North, P., Brown, D. N., \& Landstreet, J. D. 1992, A\&A, 258, 389

Pöhnl, H., Paunzen, E., \& Maitzen, H. M. 2005, A\&A, 441, 1111

Paunzen, E., \& Netopil, M. 2006, MNRAS, 371, 1641

Renson, P. 1978, A\&A, 63, 125

Renson, P., \& Manfroid, J. 2009, A\&A, 498, 961

Schaerer, D., Meynet, G., Maeder, A., \& Schaller, G. 1993a, A\&AS, 98, 523

Schaerer, D., Charbonnel, C., Meynet G., Maeder, A., \& Schaller, G. 1993b, A\&AS, 102, 339

Schaller, G., Schaerer, G., Meynet, G., \& Maeder, A. 1992, A\&AS, 96, 269

Stellingwerf, R. F. 1978, ApJ, 224, 953

Stibbs, D. W. N. 1950, MNRAS, 110, 395

Van Leeuwen, F. 2007, A\&A, 474, 653

van Leeuwen, F., Evans, D. W., Grenon, M., et al. 1997, A\&A, 323, L61

Warhurst, P. M. 2004, Baltic Astron., 13, 597

Zacharias, N., et al. 2009, UCAC3 Catalogue (VizieR On-line Data Catalog $\mathrm{I} / 315)$ 